\title{
ON ASYMPTOTIC BOROVKOV-SAKHANENKO INEQUALITY WITH UNBOUNDED PARAMETER SET
}

\author{
R. ABU-SHANAB AND A. YU. VERETENNIKOV
}

\begin{abstract}
Integral analogues of Cramér-Rao's inequalities for Bayesian parameter estimators proposed initially by Schützenberger (1958) and later by van Trees (1968) were further developed by Borovkov and Sakhanenko (1980). In this paper, new asymptotic versions of such inequalities are established under ultimately relaxed regularity assumptions and under a locally uniform nonvanishing of the prior density and with $\mathbf{R}^{1}$ as a parameter set. Optimality of Borovkov-Sakhanenko's asymptotic lower bound functional is established.
\end{abstract}

\section{INTRODUCTION}

The goal of the paper is to present new versions of Borovkov-Sakhanenko's inequalities [1, 2] also known as Cramér-Rao (CR) type integral inequalities; they may be also interpreted as lower bounds for the Bayesian mean square error. For their asympototic inequality Borovkov and Sakhanenko required Riemann integrabilty of the prior density; here only Lebesgue integrability is assumed along with some local nondegeneracy (the latter was not necessary in [1, 2]).

More precisely, first, a finite sample of size $n$, an auxiliary version of the BorovkovSakhanenko inequality (3.3), is established for unbounded parameter sets under certain relaxed conditions in comparison to [1, 2] (see Proposition 3.1). Namely, the regularity of the prior density - denoted in the sequel by $q$ - will be relaxed; instead, new conditions of nondegeneracy type will be assumed. The inequality (3.3) involves some auxiliary function, $h_{\varepsilon}$, and practically all technical issues relate to the question of how to choose this function in the best way, at least, asymptotically. Note that if the ratio $q / I$ (where $I$ is Fisher's information) were from the class $C^{1}$, then the most natural choice would have been $h_{\varepsilon}=q / I$; but if this ratio does not belong to this class, then some approximation and smoothing is required.

Second, for large samples an asymptotic version of the inequality (3.3) is of high interest and this asymptotic inequality is established also under new assumptions in Theorem 2.1. which is the main result of the paper. One more version of sufficient assumptions is presented in Theorem 2.2

Finally, in Theorem 2.3 the optimal choice of Borovkov-Sakhanenko's asymptotical functional $J$ (inverse Fisher's information integrated with the prior density) is established. This functional is strictly better, i.e., greater than the Schützenberger-van Trees

2010 Mathematics Subject Classification. Primary 62F12, 62F15.

Key words and phrases. Cramér-Rao bounds, Borovkov-Sakhanenko bounds, integral information inequalities, asymptotic efficiency.

The paper is based on a chapter in the first author's PhD Thesis [5]. Both authors are grateful to Professor Sakhanenko for his comments. The second author's work was partially supported by RFBR grant 13-01-12447 ofi_m2. 
functional, although, this remains practically unknown in the literature where asymptotic efficiency is traditionally compared with the latter under the name of van Trees alone; some comments about the names can be found in the last section of this paper.

The importance of the asymptotic Borovkov-Sakhanenko inequality for the theory may be appreciated after its comparison with the standard definition of asymptotic normality and efficiency. For many natural estimators - including Bayesian and under certain conditions maximum likelihood estimators - the asymptotic lower bound provided by the Borovkov-Sakhanenko functional is attained due to their asymptotic normality with a correct limiting variance given by inverse Fisher information. This is of a certain theoretical importance because it shows that the notion of "asymptotically optimal estimator" may be correctly defined in this way.

This paper consists of the Introduction and six further sections. Section 2 contains assumptions and main results; Section 3 contains auxiliary results; Sections 4 , 5 and 6 contain proofs of the three main results; and Section 7 is a discussion.

\section{Assumptions And main Results}

Consider a family of probability densities $\left(f(x \mid \theta), x \in \mathbf{R}^{1}\right)$ with respect to Lebesgue's measure, with a parameter $\theta \in \Theta$ where $\Theta$ is a domain in $\mathbf{R}^{1}$. In the present paper we tackle the case $\Theta=\mathbf{R}^{1}$; other unbounded cases may be treated similarly. We assume that there is a prior density $(q(\theta), \theta \in \Theta)$ and denote $f(x, \theta):=f(x \mid \theta) q(\theta)$; for the sample $X=\left(X_{1}, \ldots, X_{n}\right)$ of size $n$ of independent identically distributed random variables from the distribution $f(\cdot \mid \theta)$ denote by $L(X \mid \theta)$ the likelihood function, $L(X \mid \theta)=$ $\prod_{k=1}^{n} f\left(X_{k} \mid \theta\right)$.

Let $\theta_{n}^{*}(X)$ denote any estimator of $\theta$ where $n$ is the sample size. The quality of the estimator is assessed by the complete mean square error, i.e., by the integral

$$
\int\left(\mathrm{E}_{\theta}\left(\theta_{n}^{*}(X)-\theta\right)^{2}\right) q(\theta) d \theta \equiv \mathrm{E}\left(\theta_{n}^{*}-\theta\right)^{2},
$$

where $\mathrm{E}_{\theta}$ means expectation with respect to the density $L\left(x_{1}, \ldots, x_{n} \mid \theta\right)$ and the integration $\int \ldots d \theta$ is performed over the support of $q$, i.e., in our case over the whole line $\mathbf{R}^{1}$ (see the assumptions below). The notation $\mathrm{E}$ without the lower index $\theta$ is used for the "complete expectation", i.e., with respect to both $X$ and $\theta$.

Clearly, the estimator depends also on the sample size $n$ and in the asymptotical sense one may be interested in a lower bound for the functional

$$
\liminf _{n \rightarrow \infty} n \int\left(\mathrm{E}_{\theta}\left(\theta_{n}^{*}(X)-\theta\right)^{2}\right) q(\theta) d \theta .
$$

We assume that the derivative function $\partial L(X \mid \theta) / \partial \theta$ exists in the classical sense for each $X$ and $\theta$ (this may be slightly relaxed to a derivative in $L_{2}$ in the usual manner) and that the Fisher information function is finite,

$$
I(\theta)=\mathrm{E}_{\theta}\left(\frac{\partial \ln f\left(X_{1} \mid \theta\right)}{\partial \theta}\right)^{2}<\infty .
$$

These standard conditions are a part of the setting and will not be repeated in the main assumptions below.

The problem under consideration is lower bounds for the value (2.1). The main assumptions of the paper are as follows:

$$
0<J:=\int_{-\infty}^{\infty} \frac{q(t)}{I(t)} d t<\infty, \quad \int_{-m}^{m} \sqrt{I(t)} d t<\infty, \quad \forall m>0 .
$$


(A2) For every $m>0$ there exists $C_{m}>0$ such that

$$
C_{m}^{-1} \leq \frac{q(t)}{I(t)} \leq C_{m}, \quad-m<t<m
$$

and

$$
\inf _{t \in[-m, m]} I(t)>0, \quad \forall m>0 .
$$

As a consequence of $(\mathrm{A} 2), \inf _{t \in[-m, m]} q(t)>0$, for every $m>0$.

Remark 1. Note that continuity of $I$ is not required in (A2).

Remark 2. We do not discuss more general cases with a prior density $q$ which may vanish at some points. In such a case certain generalizations also seem possible; however, auxiliary constructions would be more involved.

Theorem 2.1. Let assumptions (A1) and (A2) be satisfied. Then,

$$
\liminf _{n \rightarrow \infty} n \mathrm{E}\left(\theta^{*}-\theta\right)^{2}=\liminf _{n \rightarrow \infty} n \int_{-\infty}^{\infty} \mathrm{E}_{t}\left(\theta^{*}-t\right)^{2} q(t) d t \geq J .
$$

Another version of assumption (A2) will be used in the next result. Note that under continuity and nondegeneracy of $I$, Riemann integrability of $q / I$ and the same integrability of $q$ are equivalent; the latter was used in [1, 2].

$\left(\mathrm{A} 2^{\prime}\right)$ Function $q / I$ is Riemann integrable on every bounded interval in $\mathbf{R}^{1}$ and

$$
\inf _{|t| \leq m} q(t)=q_{-}^{m}>0
$$

for every $m>0$ and the function $I$ is continuous.

Theorem 2.2. Let assumptions (A1) and (A2') be satisfied. Then inequality (2.2) holds true.

Theorem 2.3. Assume $\int_{-\infty}^{\infty}(q / I)(t) d t<\infty$. Then the optimal choice of $h$ in the maximization problem

$$
\frac{\left(\int_{-\infty}^{\infty} h(t) d t\right)^{2}}{\int_{-\infty}^{\infty} I(t) \frac{h^{2}(t)}{q(t)} d t} \rightarrow \sup _{h \in L_{1}(\mathbf{R})}
$$

is provided by

$$
h=c \frac{q}{I}, \quad \text { with any } c>0,
$$

and up to such a positive constant multiplier the solution of (2.3) is unique.

Remark 3. The meaning of the last theorem may be seen from the auxiliary inequality (3.3). Indeed, it follows from this inequality that its right hand side with the term $n^{-1} \int \ldots$ and with an arbitrary function $h$ (replacement of $h_{\varepsilon}$ ) dropped from the denominator may serve as a lower asymptotic bound for the left hand side. Hence, to achieve the best lower bound one should solve the maximization problem (2.3). So, Theorem 2.3 implies that the asymptotical Borovkov-Sakhanenko functional $J$ may not be improved by choosing some better auxiliary function $h$.

On the other hand, it may be noted that due to the strict Jensen's inequality, the value $J$ is, generally speaking, strictly greater than the Schützenberger-van Trees functional $\left(\int_{\mathbf{R}} I(t) q(t) d t\right)^{-1}$. 


\section{Auxiliary Results}

Let us state two technical results.

Proposition 3.1. Let $h_{\varepsilon}(t)$ be a $C^{1}$-smooth function satisfying for any $x=\left(x_{1}, \ldots, x_{n}\right)$,

$$
\lim _{t \rightarrow \pm \infty} t h_{\varepsilon}(t) L(x \mid t)=0
$$

and

$$
\int_{-\infty}^{\infty} h_{\varepsilon}(t) d t<\infty
$$

and let $\int_{-m}^{m} \sqrt{I(t)} d t<\infty$ for any $m>0$. Then,

$$
n \int_{-\infty}^{\infty} \mathrm{E}_{t}\left(\theta^{*}-t\right)^{2} q(t) d t \geq \frac{\left(\int_{-\infty}^{\infty} h_{\varepsilon}(t) d t\right)^{2}}{\int_{-\infty}^{\infty} I(t) \frac{h_{\varepsilon}^{2}(t)}{q(t)} d t+\frac{1}{n} \int_{-\infty}^{\infty} \frac{\left(h_{\varepsilon}^{\prime}(t)\right)^{2}}{q(t)} d t} .
$$

Remark 4. Practically all papers on the subject contain one or another version of this inequality, see, e.g., [1, Theorem 30.1]. However, the authors did not succeed to find in earlier works assumption (3.1), which seems to be necessary for a rigorous presentation in the case of unbounded $\Theta=\mathbf{R}^{1}$.

Proof. First, note that it suffices to prove (3.3) assuming that both the left hand side and the denominator in the right hand side are finite.

The basic identity on which the proof is based reads

$$
\mathrm{E}\left(\left(\theta^{*}(X)-\theta\right) \frac{\left(L(X \mid \theta) h_{\varepsilon}(\theta)\right)_{\theta}^{\prime}}{L(X, \theta)}\right)=\mathrm{E} \frac{h_{\varepsilon}(\theta)}{q(\theta)}=\int_{-\infty}^{\infty} h_{\varepsilon}(t) d t .
$$

Note that the left hand side in (3.4) is finite due to the Cauchy-Bouniakovsky-Schwarz inequality and the earlier assumption. In turn, (3.4) follows from

$$
\mathrm{E}\left(\left(\theta^{*}(X)-\theta\right) \frac{\left(L(X \mid \theta) h_{\varepsilon}(\theta)\right)_{\theta}^{\prime}}{L(X, \theta)}\right)=\int_{-\infty}^{\infty} \int_{-\infty}^{\infty}\left(\theta^{*}(x)-t\right)\left(L(x \mid t) h_{\varepsilon}(t)\right)_{t}^{\prime} d t d x_{1} \ldots d x_{n}
$$

Here the multiple integral with respect to $d x_{1} \ldots d x_{n}$ is denoted by a single integral symbol. Since this multiple integral converges absolutely, let us consider the internal integral

$$
\begin{aligned}
\int_{-\infty}^{\infty} & \left(\theta^{*}(x)-t\right)\left(L(x \mid t) h_{\varepsilon}(t)\right)_{t}^{\prime} d t=\lim _{M, N \rightarrow \infty} \int_{-M}^{N}\left(\theta^{*}(x)-t\right)\left(L(x \mid t) h_{\varepsilon}(t)\right)_{t}^{\prime} d t \\
& =\lim _{M, N \rightarrow \infty}\left(\left.\left(\theta^{*}(x)-t\right)\left(L(x \mid t) h_{\varepsilon}(t)\right)\right|_{t=-M} ^{t=N}-\int_{-M}^{N}\left(\theta^{*}(x)-t\right)_{t}^{\prime}\left(L(x \mid t) h_{\varepsilon}(t)\right) d t\right) \\
& =\lim _{M, N \rightarrow \infty} \int_{-M}^{N} L(x \mid t) h_{\varepsilon}(t) d t=\int_{-\infty}^{\infty} L(x \mid t) h_{\varepsilon}(t) d t .
\end{aligned}
$$

We have used explicitly (3.1). Intergating with respect to $d x_{1} \ldots d x_{n}$, we get (3.4) as required. Now the Cauchy-Bouniakovsky-Schwarz inequality applied to (3.4) gives (3.3). Proposition 3.1 is proved.

Lemma 3.2. Let assumption (A1) hold true, and let there exist a sequence

$$
0 \leq q_{m}(t) \uparrow q(t) \quad \text { (a.e.) }
$$

as $m \rightarrow \infty$, such that for any estimator $\theta^{*}$,

$$
\liminf _{n \rightarrow \infty} n \int_{-\infty}^{\infty} \mathrm{E}_{t}\left(\theta_{n}^{*}(X)-t\right)^{2} \widetilde{q}_{m}(t) d t \geq \int_{-\infty}^{\infty} \frac{\widetilde{q}_{m}(t)}{I(t)} d t,
$$


where

$$
\widetilde{q}_{m}(t)=\frac{q_{m}(t)}{\kappa_{m}} \quad \text { and } \quad \kappa_{m}=\int_{-\infty}^{\infty} q_{m}(\theta) d \theta
$$

Then,

$$
\liminf _{n \rightarrow \infty} n \mathrm{E}\left(\theta_{n}^{*}(X)-\theta\right)^{2}=\liminf _{n \rightarrow \infty} n \int_{-\infty}^{\infty} \mathrm{E}_{t}\left(\theta_{n}^{*}(X)-t\right)^{2} q(t) d t \geq J .
$$

Proof. Note that, without loss of generality, we may assume $\kappa_{m}>0$ for all $m$. The proof of the lemma follows from the monotone convergence theorem. Indeed, $\kappa_{m} \rightarrow 1$, $m \rightarrow \infty$, due to the assumption $0 \leq q_{m}(t) \uparrow q(t)$ (a.e.). So, for any $m$,

$$
\begin{aligned}
\liminf _{n \rightarrow \infty} n \int_{-\infty}^{\infty} \mathrm{E}_{t}\left(\theta_{n}^{*}(X)-t\right)^{2} q(t) d t & \geq \kappa_{m} \liminf _{n \rightarrow \infty} n \int_{-\infty}^{\infty} \mathrm{E}_{t}\left(\theta_{n}^{*}(X)-t\right)^{2} \widetilde{q}_{m}(t) d t \\
& \geq \kappa_{m} \int_{-\infty}^{\infty} \frac{\widetilde{q}_{m}(t)}{I(t)} d t=\int_{-\infty}^{\infty} \frac{q_{m}(t)}{I(t)} d t .
\end{aligned}
$$

Hence,

$$
\liminf _{n \rightarrow \infty} n \int_{-\infty}^{\infty} \mathrm{E}_{t}\left(\theta_{n}^{*}(X)-t\right)^{2} q(t) d t \geq \lim _{m \rightarrow \infty} \int_{-\infty}^{\infty} \frac{q_{m}(t)}{I(t)} d t=\int_{-\infty}^{\infty} \frac{q(t)}{I(t)} d t=J,
$$

as required. Lemma 3.2 is proved.

\section{Proof of Theorem 2.1}

1. The calculus is based on several approximations - smoothing and truncations of the function

$$
h(t):=\frac{q(t)}{I(t)}, \quad t \in \Theta=\mathbf{R} .
$$

Note that another method of smoothing was suggested in [7] in relation to some other version of the Borovkov-Sakhanenko lower bound with the analysis of the approximation errors. First, we will approximate $q$ by the appropriate $q_{m}$ and apply Lemma 3.2. Let

$$
q_{m}(t):=q(t) \mathbb{1}_{\{-m+1<t<m-1\}}, \quad m>2 .
$$

Then, $0 \leq q_{m}(t) \uparrow q(t)$, as $m \uparrow \infty$. Denote

$$
\kappa_{m}:=\int_{-m}^{m} q_{m}(\theta) d \theta \quad \text { and } \quad \widetilde{q}_{m}(t):=\frac{q_{m}(t)}{\kappa_{m}} .
$$

To prove the theorem, it suffices to show that for every $m$,

$$
\liminf _{n \rightarrow \infty} n \int_{-m}^{m} \mathrm{E}_{t}\left(\theta_{n}^{*}(X)-t\right)^{2} \widetilde{q}_{m}(t) d t \geq \int_{-m}^{m} \frac{\widetilde{q}_{m}(t)}{I(t)} d t
$$

Denote

$$
S_{m}:=\operatorname{supp}\left(q_{m}\right)=[-m+1, m-1]
$$

and

$$
h_{0, m}(t):=\frac{\widetilde{q}_{m}(t)}{I(t)} .
$$

Consider the following continuous piecewise linear function $\varphi=\varphi_{\varepsilon, m}$, with $\varepsilon \leq 1$ and $m>2$,

$$
\varphi_{\varepsilon, m}(t)= \begin{cases}(\varepsilon+1) t+\varepsilon m, & -\infty<t \leq-m+1, \\ \left(1-\frac{\varepsilon}{m-1}\right) t, & -m+1 \leq t \leq m-1, \\ (\varepsilon+1) t-\varepsilon m, & m-1 \leq t<\infty\end{cases}
$$


Notice that

$$
\begin{gathered}
\varphi_{\varepsilon, m}(-m)=-m, \quad \varphi_{\varepsilon, m}(-m+1)=-m+1+\varepsilon, \\
\varphi_{\varepsilon, m}(m-1)=m-1-\varepsilon, \quad \varphi_{\varepsilon, m}(m)=m, \\
0<\frac{1}{2} \leq \varphi_{\varepsilon, m}^{\prime} \leq 2, \quad \sup _{-\infty<t<\infty}\left|\varphi_{\varepsilon, m}^{\prime}(t)-1\right| \rightarrow 0, \quad \varepsilon \rightarrow 0, \\
\sup _{-m \leq t \leq m}\left|\varphi_{\varepsilon, m}(t)-t\right| \rightarrow 0, \quad \varepsilon \rightarrow 0,
\end{gathered}
$$

and also

$$
\widetilde{q}_{m}(-m+1-)=\widetilde{q}_{m}(m-1+)=0 .
$$

It follows from the construction of the functions $\varphi_{\varepsilon, m}$ that for any $m>2$,

$$
\sup _{-\infty<v<\infty}\left|1-\frac{1}{2 \varepsilon} \int_{\varphi_{\varepsilon, m}^{-1}(v-\varepsilon)}^{\varphi_{\varepsilon, m}^{-1}(v+\varepsilon)} d t\right| \rightarrow 0, \quad \varepsilon \rightarrow 0
$$

Let

$$
h_{\varepsilon, m}(t):=\frac{1}{2 \varepsilon} \int_{\varphi_{\varepsilon, m}(t)-\varepsilon}^{\varphi_{\varepsilon, m}(t)+\varepsilon} h_{0, m}(v) d v .
$$

Since $q_{m} \equiv 0$ outside $[-m+1, m-1]$, we conclude that

$$
h_{\varepsilon, m}(t)=0 \text { for } \varepsilon \leq 1 \text { and }|t| \geq m \text {. }
$$

Hence, the functions $h_{\varepsilon, m}$ with $\varepsilon \leq 1$ satisfy assumption (3.1).

Moreover, the function $h_{\varepsilon, m}(t)$ defined in (4.4) is absolutely continuous and differentiable almost everywhere, with a.e. (in)equalities,

$$
\begin{aligned}
h_{\varepsilon, m}^{\prime}(t) & =\frac{1}{2 \varepsilon}\left\{h_{0, m}\left(\varphi_{\varepsilon, m}(t)+\varepsilon\right)-h_{0, m}\left(\varphi_{\varepsilon, m}(t)-\varepsilon\right)\right\}, \\
\left|h_{\varepsilon, m}^{\prime}(t)\right| & \leq \frac{1}{2 \varepsilon}\left\{h_{0, m}\left(\varphi_{\varepsilon, m}(t)+\varepsilon\right)+h_{0, m}\left(\varphi_{\varepsilon, m}(t)-\varepsilon\right)\right\} .
\end{aligned}
$$

Since $q_{m} \leq q, \widetilde{q}_{m}(t)=q_{m}(t) / \kappa_{m}, q(t) / I(t) \leq C$, and $h_{0, m}(t)=\widetilde{q}_{m}(t) / I(t)$, we get,

$$
0 \leq h_{0, m}(t)=\frac{\widetilde{q}_{m}(t)}{I(t)}=\frac{q_{m}(t)}{I(t) \kappa_{m}} \leq \frac{q(t)}{I(t) \kappa_{m}} \leq \frac{C}{\kappa_{m}} .
$$

Therefore, there exists $C^{\prime}$ such that for every $\varepsilon$ small enough, and every $m$ large enough,

$$
\left|h_{\varepsilon, m}^{\prime}(t)\right| \leq \frac{C^{\prime}}{\varepsilon} \text {. }
$$

The function $h_{\varepsilon, m}(t)$ satisfies all assumptions of Proposition 3.1, so,

$$
n \int_{-m}^{m} \mathrm{E}_{t}\left(\theta^{*}-t\right)^{2} \widetilde{q}_{m}(t) d t \geq \frac{\left(\int_{-m}^{m} h_{\varepsilon, m}(t) d t\right)^{2}}{\int_{-m}^{m} I(t) \frac{h_{\varepsilon, m}^{2}(t)}{\widetilde{q}_{m}(t)} d t+\frac{1}{n} \int_{-m}^{m} \frac{\left(h_{\varepsilon, m}^{\prime}(t)\right)^{2}}{\widetilde{q}_{m}(t)} d t} .
$$

2. Let us show that

$$
\int_{-m}^{m} h_{\varepsilon, m}(t) d t \rightarrow \int_{-m}^{m} h_{0, m}(t) d t
$$

and

$$
\int_{-m}^{m} I(t) \frac{h_{\varepsilon, m}^{2}(t)}{\widetilde{q}_{m}(t)} d t \rightarrow \int_{-m}^{m} \frac{\widetilde{q}_{m}(t)}{I(t)} d t, \quad \varepsilon \rightarrow 0 .
$$

If we manage to choose $\varepsilon$ as a function of $n$ so that the term $\frac{1}{n} \int_{-m}^{m} \frac{\left(h_{\varepsilon, m}^{\prime}(t)\right)^{2}}{\widetilde{q}_{m}(t)} d t$ vanishes in the limit as $n \rightarrow \infty$, then assertions (4.6)-(4.7) would imply (4.5) with $h_{\varepsilon, m}$ replaced by $h_{m}$, which would allow us to apply Lemma 3.2 . 
3. Recall that $h_{0, m} \equiv 0$ outside the interval $[-m+1, m-1]$, so that

$$
\int_{-m+\varepsilon}^{m-\varepsilon} h_{0, m}(t) d t=\int_{-m}^{m} h_{0, m}(t) d t
$$

for any $\varepsilon \leq 1$. Because of this and by virtue of (4.3) and Lebesgue's dominated convergence theorem and assuming that always $\varepsilon \leq 1$, we have

$$
\begin{aligned}
\int_{-m}^{m} h_{\varepsilon, m}(t) d t & =\int_{-m}^{m} d t \frac{1}{2 \varepsilon} \int_{\varphi_{\varepsilon, m}(t)-\varepsilon}^{\varphi_{\varepsilon, m}(t)+\varepsilon} h_{0, m}(v) d v=\int_{-m+\varepsilon}^{m-\varepsilon} h_{0, m}(v) d v \frac{1}{2 \varepsilon} \int_{\varphi_{\varepsilon, m}^{-1}(v-\varepsilon)}^{\varphi_{\varepsilon, m}^{-1}(v+\varepsilon)} d t \\
& =\int_{-m}^{m} \mathbb{1}_{\{-m+\varepsilon<v<m-\varepsilon\}} h_{0, m}(v) d v \frac{1}{2 \varepsilon} \int_{\varphi_{\varepsilon, m}^{-1}(v-\varepsilon)}^{\varphi_{\varepsilon, m}^{-1}(v+\varepsilon)} d t \\
& \rightarrow \int_{-m}^{m} h_{0, m}(v) d v, \quad \varepsilon \rightarrow 0 .
\end{aligned}
$$

So, the convergence (4.6) holds true.

4. To show (4.7), we notice that

$$
\begin{aligned}
\int_{-m}^{m} & \frac{I(t)}{\widetilde{q}_{m}(t)}\left(h_{\varepsilon, m}^{2}(t)-h_{0, m}^{2}(t)\right) d t \\
\quad & =\int_{-m}^{m} \frac{I(t)}{\widetilde{q}_{m}(t)}\left(h_{\varepsilon, m}(t)-h_{0, m}(t)\right)\left(h_{\varepsilon, m}(t)+h_{0, m}(t)\right) d t .
\end{aligned}
$$

Since the terms $I(t) / \widetilde{q}_{m}(t)$ and $\left(h_{\varepsilon, m}(t)+h_{0, m}(t)\right)$ are uniformly bounded on $S_{m}$, it suffices to establish the convergence

$$
\int_{-m}^{m}\left|h_{\varepsilon, m}(t)-h_{0, m}(t)\right| d t \rightarrow 0, \quad \varepsilon \rightarrow 0 .
$$

Let $\delta>0$ and, given $m$, let us approximate the function $h_{0, m}(t)$ in $L_{1}[-m, m]$ by some continuous function $h_{0, m}^{\delta}(t)$, which equals identically zero outside the intevral $[-m, m]$, so that

$$
\int_{-m}^{m}\left|h_{0, m}(t)-h_{0, m}^{\delta}(t)\right| d t<\delta
$$

This is, clearly, possible, since the space $C[-m, m]$ is a dense subspace in $L_{1}[-m, m]$. Then, assuming that $\varepsilon>0$ is so small that the left hand side in (4.3) does not exceed the value 1 (recall that it actually tends to zero with $\varepsilon$ ) and denoting

$$
h_{\varepsilon, m}^{\delta}(t):=\frac{1}{2 \varepsilon} \int_{\varphi_{\varepsilon, m}(t)-\varepsilon}^{\varphi_{\varepsilon, m}(t)+\varepsilon} h_{0, m}^{\delta}(v) d v,
$$

we get

$$
\begin{aligned}
\int_{-m}^{m}\left|h_{\varepsilon, m}(t)-h_{\varepsilon, m}^{\delta}(t)\right| d t & =\int_{-m}^{m}\left|\frac{1}{2 \varepsilon} \int_{\varphi_{\varepsilon, m}(t)-\varepsilon}^{\varphi_{\varepsilon, m}(t)+\varepsilon}\left(h_{0, m}(v)-h_{0, m}^{\delta}(v)\right) d v\right| d t \\
& \leq \int_{-m}^{m} \frac{1}{2 \varepsilon} \int_{\varphi_{\varepsilon, m}(t)-\varepsilon}^{\varphi_{\varepsilon, m}(t)+\varepsilon}\left|h_{0, m}(v)-h_{0, m}^{\delta}(v)\right| d v d t \\
& =\int_{-m}^{m}\left|h_{0, m}(v)-h_{0, m}^{\delta}(v)\right| d v \frac{1}{2 \varepsilon} \int_{\varphi_{\varepsilon, m}^{-1}(v-\varepsilon)}^{\varphi_{\varepsilon, m}^{-1}(v+\varepsilon)} d t \leq 2 \delta
\end{aligned}
$$


Hence, for the values of $\varepsilon$ satisfying the above,

$$
\begin{aligned}
\int_{-m}^{m}\left|h_{\varepsilon, m}(t)-h_{0, m}(t)\right| d t \leq & \int_{-m}^{m}\left|h_{\varepsilon, m}(t)-h_{\varepsilon, m}^{\delta}(t)\right| d t+\int_{-m}^{m}\left|h_{0, m}(t)-h_{0, m}^{\delta}(t)\right| d t \\
& +\int_{-m}^{m}\left|h_{\varepsilon, m}^{\delta}(t)-h_{0, m}^{\delta}(t)\right| d t \\
\leq & 2 \delta+\delta+\int_{-m}^{m}\left|h_{\varepsilon, m}^{\delta}(t)-h_{0, m}^{\delta}(t)\right| d t .
\end{aligned}
$$

For every fixed $\delta>0$, the latter integral tends to zero as $\varepsilon \rightarrow 0$, because the function $h_{0, m}^{\delta}(t)$ is uniformly continuous, and due to the convergence

$$
\sup _{t}\left|h_{\varepsilon, m}^{\delta}(t)-h_{0, m}^{\delta}(t)\right| d t \rightarrow 0, \quad \varepsilon \rightarrow 0,
$$

by virtue of (4.3) and (4.9). Therefore, for every $\delta>0$,

$$
\limsup _{\varepsilon \rightarrow 0} \int_{-m}^{m}\left|h_{\varepsilon, m}(t)-h_{0, m}(t)\right| d t \leq 3 \delta .
$$

However, the left hand side here does not depend on $\delta$, hence, (4.8) holds true, which implies (4.7).

5. From (4.5), (4.6) and (4.7) we conclude that

$$
\liminf _{n \rightarrow \infty} n \int_{-m}^{m} \mathrm{E}_{t}\left(\theta^{*}-t\right)^{2} \widetilde{q}_{m}(t) d t \geq \frac{\left(\int_{-m}^{m} h_{0, m}(t) d t\right)^{2}}{\int_{-m}^{m} h_{0, m}(t) d t+\lim _{\sup } \operatorname{sum}_{n \rightarrow \infty} \frac{1}{n} \int_{-m}^{m} \frac{\left(h_{\varepsilon, m}^{\prime}(t)\right)^{2}}{\widetilde{q}_{m}(t)} d t} .
$$

We estimate,

$$
\frac{1}{n} \int_{-m}^{m} \frac{\left(h_{\varepsilon, m}^{\prime}(t)\right)^{2}}{\widetilde{q}_{m}(t)} d t \leq \frac{1}{n} \int_{-m}^{m} \frac{C^{\prime 2}}{\varepsilon^{2} \widetilde{q}_{m}(t)} d t=\frac{C^{\prime 2}}{\varepsilon^{2} n} \int_{-m}^{m} \frac{1}{\widetilde{q}_{m}(t)} d t .
$$

For any fixed $m$, we can choose $\varepsilon=\varepsilon(n)=C^{\prime} n^{-1 / 5}$, so that $\lim _{n \rightarrow \infty} 1 /\left(\varepsilon^{2} n\right)=0$. Hence, we obtain

$$
\liminf _{n \rightarrow \infty} n \int_{-m}^{m} \mathrm{E}_{t}\left(\theta^{*}-t\right)^{2} \widetilde{q}_{m}(t) d t \geq \int_{-m}^{m} h_{0, m}(t) d t .
$$

Since

$$
h_{0, m}(t)=\frac{\widetilde{q}_{m}(t)}{I(t)}
$$

the desired asymptotic inequality (2.2) follows due to Lemma 3.2 . Theorem 2.1] is proved.

\section{Proof of Theorem 2.2}

1. Let us denote

$$
q_{-}^{m}:=\inf _{-m \leq t \leq m} q(t)>0 ;
$$

see assumption $\left(\mathrm{A} 2^{\prime}\right)$. As in the proof of Theorem 2.1. we will approximate $q$ by an appropriate $\tilde{q}_{m}$ and apply Lemma 3.2. Let

$$
\begin{gathered}
q_{m}(t):=q(t) \mathbb{1}_{\{-m+1<t<m-1\}}, \quad m>1, \\
\kappa_{m}=\int_{-m}^{m} q_{m}(\theta) d \theta \quad \text { and } \quad \widetilde{q}_{m}(t)=\frac{q_{m}(t)}{\kappa_{m}} .
\end{gathered}
$$

To prove the theorem, it suffices to show that for every $m$,

$$
\liminf _{n \rightarrow \infty} n \int_{-m}^{m} \mathrm{E}_{t}\left(\theta_{n}^{*}(X)-t\right)^{2} \widetilde{q}_{m}(t) d t \geq \int_{-m}^{m} \frac{\widetilde{q}_{m}(t)}{I(t)} d t .
$$


For the function $\int_{-m}^{m}\left(h_{\varepsilon}^{\prime}(t)\right)^{2} / \tilde{q}_{m}(t) d t$, the following notation will be used:

$$
H_{m}(\varepsilon):=\int_{-m}^{m}\left(h_{\varepsilon}^{\prime}(t)\right)^{2} / \tilde{q}_{m}(t) d t .
$$

Let

$$
\begin{gathered}
h_{0, m}(t):=\tilde{q}_{m}(t) / I(t), \\
\bar{h}_{\varepsilon, m}(t):=\min _{|u| \leq \varepsilon} \frac{\tilde{q}_{m}(t+u)}{I(t+u)}, \quad \tilde{h}_{\varepsilon, m}(t):=\bar{h}_{\varepsilon, m}(t) \wedge \frac{q_{-}^{m}}{\varepsilon}, \quad-m \leq t \leq m,
\end{gathered}
$$

and

$$
h_{\varepsilon, m}(t):=\frac{1}{2 \varepsilon} \int_{t-\varepsilon}^{t+\varepsilon} \tilde{h}_{\varepsilon, m}(v) d v .
$$

With this definition, we clearly have

$$
\tilde{h}_{\varepsilon, m}(t) \leq h_{0, m}(t) \quad \text { and } \quad 0 \leq h_{\varepsilon, m}(t) \leq h_{0, m}(t) .
$$

Now, the function $h_{\varepsilon, m}$ defined in (5.2) is absolutely continuous and differentiable almost everywhere, with

$$
\left|h_{\varepsilon, m}^{\prime}(t)\right| \leq \frac{C \tilde{q}_{m}(t)}{\varepsilon} \wedge \frac{\tilde{q}_{m}(t)}{I(t)},
$$

and $h_{\varepsilon, m}(-m)=h_{\varepsilon, m}(m)=0$, for any $\varepsilon>0$. Due to assumption (A2'), the function $H_{m}(\varepsilon)$ is finite and, moreover,

$$
H_{m}(\varepsilon) \leq \frac{C}{\varepsilon^{2}} \int_{-m}^{m} \frac{\tilde{q}_{m}^{2}(t)}{\tilde{q}_{m}(t)} d t \leq \frac{C}{\varepsilon^{2}}
$$

2. Let us show that

$$
\tilde{h}_{\varepsilon, m}(t) \rightarrow h_{0, m}(t), \quad \varepsilon \downarrow 0 \quad \text { (a.e.). }
$$

Thus, due to the Lebesgue dominated convergence theorem, it suffices to show that

$$
\int_{-m}^{m}\left(h_{0, m}(t)-\tilde{h}_{\varepsilon, m}(t)\right) d t \downarrow 0, \quad \varepsilon \downarrow 0 .
$$

This follows similarly to [1, Proof of Theorem 30.5], where this hint is applied to the function $q$. We have, by virtue of the Riemann integrability condition and of the theorem about convergence of Darboux integral sums,

$$
\begin{gathered}
\sum_{k} \bar{h}_{\delta, m}(2 k \delta) 2 \delta \rightarrow \int_{-m}^{m} h_{0, m}(t) d t, \quad \delta \rightarrow 0, \\
\sum_{k} \bar{h}_{\delta, m}((2 k+1) \delta) 2 \delta \rightarrow \int_{-m}^{m} h_{0, m}(t) d t, \quad \delta \rightarrow 0 .
\end{gathered}
$$

Let us estimate the difference,

$$
0 \leq \sum_{k}\left(\bar{h}_{\delta, m}(2 k \delta)-\tilde{h}_{\delta, m}(2 k \delta)\right) 2 \delta \leq 2 \delta \sum_{k} \bar{h}_{\delta, m}(2 k \delta) \mathbb{1}_{\left\{\bar{h}_{\delta, m}(2 k \delta)>q_{-}^{m} /(2 \delta)\right\}} .
$$

Since $h_{0, m}$ is Riemann integrable, it must be bounded on $[-m, m]$, and so is $\bar{h}_{\delta, m} \leq h_{0, m}$. Since $\inf _{t \in[-m, m]} \tilde{q}_{m}(t)>0$, it follows from $\left(\mathrm{A} 2^{\prime}\right)$ that $\tilde{h}_{\delta, m} \equiv \bar{h}_{\delta, m}$ as $\delta$ is small enough. Then, of course,

$$
\mathbb{1}_{\left\{\bar{h}_{\delta, m}(2 k \delta)>q_{-}^{m} /(2 \delta)\right\}}=0 .
$$

Therefore, the sum $\sum_{k} \bar{h}_{\delta, m}(2 k \delta) \mathbb{1}_{\left\{\bar{h}_{\delta, m}(2 k \delta)>q_{-}^{m} /(2 \delta)\right\}}$ equals zero if $\delta$ is small enough. So,

$$
0 \leq \sum_{k}\left(\bar{h}_{\delta, m}(2 k \delta)-\tilde{h}_{\delta, m}(2 k \delta)\right) 2 \delta \rightarrow 0, \quad \delta \rightarrow 0 .
$$


Similarly,

$$
0 \leq \sum_{k}\left(\bar{h}_{\delta, m}((2 k+1) \delta)-\tilde{h}_{\delta, m}((2 k+1) \delta)\right) 2 \delta \rightarrow 0, \quad \delta \rightarrow 0 .
$$

Hence,

$$
\begin{aligned}
\int_{-m}^{m} \tilde{h}_{\varepsilon, m}(t) d t & \geq\left(\sum_{k} \tilde{h}_{2 \varepsilon, m}(4 k \varepsilon) 2 \varepsilon+\sum_{k} \tilde{h}_{2 \varepsilon, m}((4 k+2) \varepsilon) 2 \varepsilon\right) \\
& \rightarrow \int_{-m}^{m} h_{0, m}(t) d t, \quad \varepsilon \rightarrow 0 .
\end{aligned}
$$

Since $\int_{-m}^{m} \tilde{h}_{\varepsilon, m} \leq \int_{-m}^{m} h_{0, m}$, the latter convergence implies (5.5). Notice that, strictly speaking, so far we have shown just the convergence

$$
\int_{-m}^{m}\left(h_{0, m}(t)-\tilde{h}_{\varepsilon, m}(t)\right) d t \rightarrow 0, \quad \varepsilon \downarrow 0,
$$

which may be monotone or not. But, by construction, the function $\tilde{h}_{\varepsilon, m}$ increases with $\varepsilon$ decreasing. This implies (5.5). Hence, (5.4) holds true almost everywhere on $-m \leq$ $t \leq m$.

3. Notice that $h_{\varepsilon, m}$ satisfies the assumptions of Proposition 1, being differentiable and since it vanishes at $-m$ and $m$. So, we get, with $\varepsilon=(C n)^{-1 / 3}$,

$$
n \int_{-m}^{m} \mathrm{E}_{\theta}\left(\theta_{n}^{*}-\theta\right)^{2} \tilde{q}_{m}(\theta) d \theta \geq \frac{\left(\int_{-m}^{m} h_{\varepsilon, m}(t) d t\right)^{2}}{\int_{-m}^{m} I(t) h_{\varepsilon, m}(t)^{2} / \tilde{q}_{m}(t) d t+n^{-1 / 3}} .
$$

Hence, to complete the proof, it suffices to establish

$$
\int_{-m}^{m} h_{\varepsilon, m}(t) d t \rightarrow \int_{-m}^{m} h_{0, m}(t) d t
$$

and

$$
\int_{-m}^{m} \frac{I(t) h_{\varepsilon, m}(t)^{2}}{\tilde{q}_{m}(t)} d t \rightarrow \int_{-m}^{m} \frac{\tilde{q}_{m}(t)}{I(t)} d t \equiv \int_{-m}^{m} \frac{I}{\tilde{q}_{m}} h_{0, m}^{2} d t, \quad \varepsilon \rightarrow 0 .
$$

4. We have

$$
\begin{aligned}
0 & \leq \int_{-m}^{m}\left(h_{0, m}(t)-h_{\varepsilon, m}(t)\right) d t=\int_{-m}^{m}\left(h_{0, m}(t)-\frac{1}{2 \varepsilon} \int_{t-\varepsilon}^{t+\varepsilon} \tilde{h}_{\varepsilon, m}(v) d v\right) d t \\
& =\int_{-m}^{m} \frac{1}{2 \varepsilon} \int_{t-\varepsilon}^{t+\varepsilon}\left(h_{0, m}(t)-\tilde{h}_{\varepsilon, m}(v)\right) d v d t \\
& =\int_{-m}^{m} \frac{1}{2 \varepsilon} \int_{t-\varepsilon}^{t+\varepsilon}\left(h_{0, m}(t)-\tilde{h}_{\varepsilon, m}(t)\right) d v d t+\int_{-m}^{m} \frac{1}{2 \varepsilon} \int_{t-\varepsilon}^{t+\varepsilon}\left(\tilde{h}_{\varepsilon, m}(t)-\tilde{h}_{\varepsilon, m}(v)\right) d v d t .
\end{aligned}
$$

Here,

$$
\int_{-m}^{m} \frac{1}{2 \varepsilon} \int_{t-\varepsilon}^{t+\varepsilon}\left(h_{0, m}(t)-\tilde{h}_{\varepsilon, m}(t)\right) d v d t=\int_{-m}^{m}\left(h_{0, m}(t)-\tilde{h}_{\varepsilon, m}(t)\right) d t \rightarrow 0, \quad \varepsilon \rightarrow 0,
$$

due to (5.5). On the other hand,

$$
\begin{aligned}
\int_{-m}^{m} & \frac{1}{2 \varepsilon} \int_{t-\varepsilon}^{t+\varepsilon}\left(\tilde{h}_{\varepsilon, m}(t)-\tilde{h}_{\varepsilon, m}(v)\right) d v d t \\
& =\int \tilde{h}_{\varepsilon, m}(t) d t-\int \tilde{h}_{\varepsilon, m}(v)\left(\frac{1}{2 \varepsilon} \int_{v-\varepsilon}^{v+\varepsilon} 1 d t\right) d v=0 .
\end{aligned}
$$

Thus, indeed, (5.8) holds true. 
5. Further, by virtue of (5.3) and (5.8), we also have,

$$
\begin{aligned}
0 & \leq \int \frac{I(t)}{\tilde{q}_{m}(t)}\left(h_{0, m}^{2}(t)-h_{\varepsilon, m}^{2}(t)\right) d t=\int h_{0, m}^{-1}\left(h_{0, m}(t)-h_{\varepsilon, m}(t)\right)\left(h_{0, m}(t)+h_{\varepsilon, m}(t)\right) d t \\
& \leq \int h_{0, m}^{-1}\left(h_{0, m}(t)-h_{\varepsilon, m}(t)\right) 2 h_{0, m}(t) d t=2 \int\left(h_{0, m}(t)-h_{\varepsilon, m}(t)\right) d t \rightarrow 0, \quad \varepsilon \rightarrow 0 .
\end{aligned}
$$

This shows (5.9). Now, from (5.7), (5.8) and (5.9) the desired inequality (4.2) follows. By virtue of Lemma 3.2 this finally implies (2.2). Theorem 2.2 is proved.

\section{Proof of Theorem 2.3}

1. First, we show that considering $h$, which might change sign, may not give a better bound. To this aim, we note that the maximization problem (2.3), may be presented as an equivalent maximization problem,

$$
h: \int_{-\infty}^{\infty} \max _{(t) \frac{h^{2}(t)}{q(t)} d t=1} \int_{-\infty}^{\infty} h(t) d t .
$$

It is clear that allowing to change sign for $h$ from positive to negative at some points may not increase the value of the integral $\int_{-\infty}^{\infty} h(t) d t$. In other words, the global maximizer $h$ in the problem (6.1) may be, indeed, only nonnegative.

2. For any $h \not \equiv 0$ by the Cauchy-Bouniakovsky-Schwarz inequality, we have

$$
\frac{\left(\int_{-\infty}^{\infty} h(t) d t\right)^{2}}{\int_{-\infty}^{\infty} I(t) \frac{h^{2}(t)}{q(t)} d t} \leq \int_{-\infty}^{\infty} \frac{q(t)}{I(t)} d t
$$

since

$$
\left(\int_{-\infty}^{\infty} h(t) d t\right)^{2} \leq \int_{-\infty}^{\infty} \frac{q}{I}(t) d t \times \int_{-\infty}^{\infty} I(t) \frac{h^{2}(t)}{q(t)} d t .
$$

On the other hand, if we choose $h=c q / I$ (with any $c>0$ ), then

$$
\frac{\left(\int_{-\infty}^{\infty} h(t) d t\right)^{2}}{\int_{-\infty}^{\infty} I(t) \frac{h^{2}(t)}{q(t)} d t}=\frac{c^{2}\left(\int q / I(t) d t\right)^{2}}{\int(I / q) c^{2}(q / I)^{2}(t) d t}=\int_{-\infty}^{\infty} q(t) / I(t) d t
$$

The equality in (6.2) is only possible for the choice of $h$ where $I(t) \frac{h^{2}(t)}{q(t)}=$ const $\times q / I$, by virtue of the equality part of the Cauchy-Bouniakovsky-Schwarz inequality. The latter equation implies that necessarily $|h|=c q / I$. Since optimal $h$ should be nonnegative, this means that the optimal choice is, indeed, provided by (2.4) and Theorem 2.3 is proved.

\section{Discussion}

In this little section some less known issues about the Schützenberger-van Trees and the Borovkov-Sakhanenko lower bounds will be discussed. For a long time it was common knowledge that integral CR bounds were introduced in [9]. In fact, more than ten years earlier an estimate of this type with a short but rigorous proof was published in [8. It may be interesting to note that in $[8]$ CR bounds are called Fréchet-Cramér bounds (see [3]). Hence, it would be more than appropriate to call such bounds, as a minimum, by two names, Schützenberger-van Trees. These bounds are in use in the literature in the issues of asymptotical efficiency of estimators (see, for example, 4]). Nevertheless, as was emphasized earlier in the paper more precise asymptotical bounds are provided by the Borovkov-Sakhanenko inequality. 
Next, note that any limiting assertion becomes more useful if some rate of convergence is established. In this sense, Borovkov and Sakhanenko's results required some complementary bounds of remainder terms. Under certain additional smoothness, such bounds have been established in [1, 2, 6. However, even without convergence rate, a limiting assertion may be helpful as such since it shows asymptotical properties of estimators under less restrictive conditions in comparison to what is needed for evaluating errors. Here results like Theorems 2.1 or 2.2 above may be of some help.

Finally, note that in 10] some development of the Borovkov-Sakhanenko inequalities was presented for a bounded parameter set. As it turns out, unboundedness of this set requires a lot more technical work and additional assumptions to tackle this unboundedness.

\section{BIBLIOGRAPHY}

1. A. A. Borovkov, Mathematical Statistics, Gordon and Breach, Amsterdam, 1998. MR1712750 (2000f:62003)

2. A. A. Borovkov and A. I. Sakhanenko, Estimates for averaged quadratic risk, Probab. Math. Statist. 1 (1980), no. 2, 185-195. (Russian) MR626310(84a:62034)

3. M. Fréchet, Sur l'extension de certaines évaluations statistiques au cas de petits échantillons, Rev. Inst. Internat. Statist. 11 (1943), 182-205.

4. R. D. Gill and B. Y. Levit, Applications of the van Trees inequality: a Bayesian Cramér-Rao bound, Bernoulli 1 (1995), 59-79. MR1354456 (97c:62051)

5. Reman Abu-Shanab, Information Inequalities and Parameter Estimation, PhD Thesis, University of Leeds, UK, 2009.

6. A. E. Shemyakin, Rao-Cramér type integral inequalities for the estimates of a vector parameter, Theory Probab. Appl. 33 (1985) no. 3, 426-434.

7. A. E. Shemyakin, Rao-Cramér type multidimensional integral inequalities for parametric families with singularities, Sib. Math. J. (1992), 706-715. MR1142080 (93d:62047)

8. M. P. Schützenberger, A propos de l'inégalité de Fréchet-Cramér, Publ. Inst. Statist. Univ. Paris 7 (1958), no. 3/4, 3-6; http://igm.univ-mlv.fr/ berstel/Mps/Travaux/A/ 1958FrechetInstStat.pdf MR0105762 (21:4498)

9. H. van Trees, Detection, Estimation and Modulation Theory, vol. I, Wiley, New York, 1968.

10. A. Yu. Veretennikov, On asymptotic information integral inequalities, Theory Stoch. Process. 13(29) (2007), no. 1-2, 294-307. MR2343831 (2008m:62050)

P.O. Box 32038, Department of Mathematics, College of Science, University of Bahrain, KINGDOM OF BAHRAIN

E-mail address: raboshanab@uob.edu.bh

School of Mathematics, University of Leeds, LS2 9JT, United Kingdom \& Institute for Information Transmission Problems, Moscow, Russia - and - National Research University Higher School of Economics, Moscow, Russia

E-mail address: a.veretennikov@leeds.ac.uk 